\title{
In Silico and In Vitro Investigation of the Piperine's Male Contraceptive Effect: Docking and Molecular Dynamics Simulation Studies in Androgen-Binding Protein and Androgen Receptor
}

Authors

Affiliations
Gopichand Chinta ${ }^{1 *}$, Mariasoosai Ramya Chandar Charles ${ }^{2 *}$, Ivana Klopčič ${ }^{3}$, Marija Sollner Dolenc ${ }^{3}$, Latha Periyasamy ${ }^{4}$, Mohane Selvaraj Coumar ${ }^{2}$

The affiliations are listed at the end of the article

\author{
Key words \\ - androgen-binding protein \\ - androgen receptor \\ - docking \\ - male contraceptive \\ - molecular dynamics \\ simulation \\ - piperine
}

\section{Abstract \\ $\nabla$}

Understanding the molecular mechanism of action of traditional medicines is an important step towards developing marketable drugs from them. Piperine, an active constituent present in the Piper species, is used extensively in Ayurvedic medicines (practiced on the Indian subcontinent). Among others, piperine is known to possess a male contraceptive effect; however, the molecular mechanism of action for this effect is not very clear. In this regard, detailed docking and molecular dynamics simulation studies of piperine with the androgen-binding protein and androgen receptors were carried out. Androgen receptors control male sexual behavior and fertility, while the androgen-binding protein binds testosterone and maintains its concentration at optimal levels to stimulate spermatogenesis in the testis. It was found that piperine docks to the androgen-binding protein, similar to dihydrotestosterone, and to androgen receptors, similar to cyproterone acetate (antagonist). Also, the piperine-androgen-binding protein and piperine-androgen receptors interactions were found to be stable throughout 30 ns of molecular dynamics simulation. Further, two independent simulations for $10 \mathrm{~ns}$ each also confirmed the stability of these interactions. Detailed analysis of the piperine-androgen-binding protein interactions shows that

\section{Introduction}

\section{$\nabla$}

Since the beginning of human civilization, natural products have been a source for several medicinal agents. Their use in the treatment and prevention of diseases has been recorded in ancient medical literatures, including that of Ayurveda (practiced

\footnotetext{
* These authors equally contributed to this work.
}

piperine interacts with Ser42 of the androgenbinding protein and could block the binding with its natural ligands dihydrotestosterone/testosterone. Moreover, piperine interacts with Thr577 of the androgen receptors in a manner similar to the antagonist cyproterone acetate. Based on the in silico results, piperine was tested in the MDA-kb2 cell line using the luciferase reporter gene assay and was found to antagonize the effect of dihydrotestosterone at nanomolar concentrations. Further detailed biochemical experiments could help to develop piperine as an effective male contraceptive agent in the future.

\section{Abbreviations}

$\nabla$

ABP: androgen-binding protein

AR: androgen receptor

CPA: cyproterone acetate

DHT: dihydrotestosterone

MD: molecular dynamics

RMSDs: root mean square deviations

RMSFs: root mean square fluctuations

SBHG: sex hormone-binding globulin

TCM: traditional Chinese medicine

Supporting information available online at http://www.thieme-connect.de/products

on the Indian subcontinent) and TCM. From the past century, exploration and exploitation of this traditional medicinal knowledge using modern scientific tools have resulted in identifying the bioactive constituents and in the development of several of them as drugs for treating diseases [1$3]$. For example, the modern drugs quinine (antimalarial), aspirin (anti-inflammatory analgesic), vincristine (anticancer), artemisinin (antimalarial), and morphine (analgesic) have their basis 


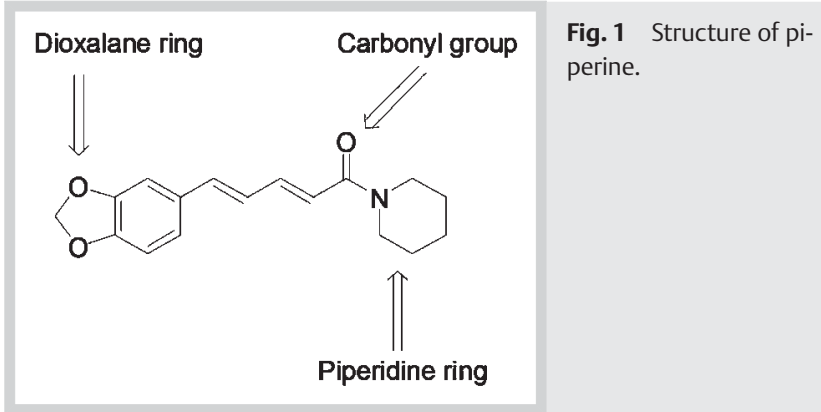

from traditional medicines [4]. Currently, natural products and their derivatives contribute to $50 \%$ of all drugs in clinical use. This highlights the potential use of traditional medicinal knowledge for developing modern medicines. Considering the difficulty, money, and time involved in discovering and developing a drug, traditional medicinal plants are an ideal knowledge base for new drug development.

In this regard, several herbal drugs used in Ayurveda have been explored to identify their bioactive constituents and develop them as drugs. Piper longum L. (long pepper) and Piper nigrum L. (black pepper) are two such plants used in Ayurvedic medicine [5-7]. Piperine ( Fig. 1) is an active constituent present in both plants, and is responsible for the pungency of these herbs $[5,6]$. In preclinical studies, piperine proved to be effective in treating various disorders like depression and cognitive difficulties [8,9]. Also, piperine has been proven to have antiplatelet [10], antithyroid [11], hepatoprotective [12], antidiabetic [13], anti-inflammatory [14], antitumor [15], antiasthmatic [16], antileishmanial [17], antimutagenic [18], and antidiarrheal activities [19].

More interestingly, many studies had now proven the bioenhancing properties of piperine when simultaneously administered with curcumin [20], gallic acid [21], tifferon [22], $\beta$-carotene [23], and (-) epigallatocatechin-3-gallate [24]. Piperine also enhances the bioavailability of drugs like tetracyclines, vasicine, sulfadiazine, rifampicin, pyrazinamide, isoniazid, ethambutol, phenobarbitone, phenytoin, carbamazepine, nimesulide, indomethacin, ciprofloxacin, and dapsone $[25,26]$. Based on the bioavailability-enhancing property of piperine, formulations containing piperine $(10 \mathrm{mg})$, rifampicin $(200 \mathrm{mg})$, and isoniazid $(300 \mathrm{mg}$ ) have been marketed in India since 2009 to treat tuberculosis [27]. Further, a literature survey of medicinal plants conducted in 2006 identified the Piper species to possess male contraceptive activity [28]. As the use of condoms or a vasectomy is the only choice available for male contraception, interest among researchers to investigate the possibility to develop piperine-based male contraception has grown in the last decade. For this, the effect of piperine on the rat testis, epididymis, and testicular apoptosis was studied in detail by various researchers [29-32]. However, a detailed understanding of the molecular mechanism of action of piperine's male contraceptive activity is still lacking.

In males, expression of AR is very high in the tissues of urogenital organs and the prostate [33]. The androgens, testosterone, and DHT are the main ligands for AR. Recent evidences suggest that AR function is required in Sertoli cells for the terminal differentiation of haploid spermatids [34] and the AR in Leydig cells and peritubular myoid cells is essential for spermatogenesis [35]. Hence, it is clear that normal AR function is essential to develop male phenotype, and it also controls male sexual behavior, the maintenance of spermatogenesis, and fertility [36,37]. ARs be- longs to the nuclear receptor super family and has four main domains $[38,39]$ : the amino-terminal activation domain (TAD), the DNA-binding domain, the hinge domain, and the ligand-binding domain (LBD). The receptor is activated when androgen binds to the C-terminal LBD, triggering a cascade of molecular events, including interactions between LBD and TAD, and the recruitment of transcriptional coactivators. Immediate to the binding of androgens to AR, translocation of AR to the nucleus occurs, which results in transcriptional regulation of androgen target genes $[38,39]$.

ABP usually secretes into the lumen of the somniferous tubule and into the interstitial fluid around the spermatogenic cell environment by the combined effect of a follicle stimulating hormone and testosterone on the Sertoli cells. The ABP binds to testosterone and maintains its concentration at optimal levels to stimulate the spermatogenesis [40]. ABP is a differently glycosylated isoform of SBHG. Human SBHG is a homodimer with 373 amino acids. The steroid-binding site is composed of residues from eight beta strands, including Ser42, Phe56, Gly58, Asp65, Phe67, Asn82, Met139, Val112, and Leu171, which are conserved among all the SBHGs. Mutagenesis studies show that replacing Ser42 with a lysine leads to the loss of steroid binding to human SBHG expressed in $\mathrm{CHO}$ cells, suggesting it to be an important residue for steroid binding [41].

Considering the importance of AR and ABP as potential targets for male contraception, we planned to investigate the possible interaction of piperine with these targets. To our knowledge, no in silico study was conducted to investigate the interactions of piperine with $A R$ and $A B P$. The present study was aimed to ascertain the interaction of piperine with AR and ABP by docking and molecular dynamics simulation studies. This could help in better understanding the molecular mechanism of piperine's male contraceptive effect.

\section{Results and Discussion}

To investigate the potential of piperine to interact with $A B P$ and $A R$, the 3D protein structures PDB ID: 1D2S (ABP) and PDB ID: 1E3G (AR) were used for the docking studies. Initially, the ligands (DHT in ABP; metribolone as the agonist and CPA as the antagonist in AR) present in the crystal structures were docked to their respective protein in order to validate the docking protocol. Docking of the cocrystallized ligands to their respective proteins would help in knowing if the docking algorithm used by the LibDock module is able to reproduce the bioactive conformation accurately. The ability to identify the correct bioactive conformation by the docking algorithm not only acts as a validation step, but also acts as a measure of reliability of the docking results of the new ligands under investigation.

Docking of DHT to ABP produced 62 docked poses with the topdocking pose having a LibDock score of 108.14 ( Table 1 ). The top docked pose of DHT to ABP is very similar to that of the crystallographic conformation, as measured by the RMSD ( $3.4 \AA$ ) between the docked pose and the crystallographic conformation. The docked pose of DHT made two H-bonds with Ser42 ( Fig. 2A); however, two other H-bond interactions with Asn82 \& Asp65, observed in the crystallographic conformation, were not observed in the docked pose.

Docking of metribolone with AR showed that the ligand could bind to the target protein with a LibDock score of 112.31 ( Table 1). The docked conformation of metribolone was very similar to 
Table 1 Comparison of the docking interactions of piperine and native ligands in ABP and AR.

\begin{tabular}{lllll}
\hline Target protein & Ligand & LibDock score & No. of H-bonds & H-bond forming residue (distance in Å) \\
\hline ABP (1D2S) & DHT (Crystal structure) & - & 5 & $\begin{array}{l}\text { SER42 (2.76 and 3.09), ASP65 (2.73 and 2.72), ASN82 } \\
\text { (2.93) }\end{array}$ \\
& & & & SER42 (2.18 and 3.11) \\
& DHT (Docked pose) & 108.14 & 2 & SER42 (2.67), TRP66 (2.94), ASN82 (2.72) \\
& Piperine (Docked pose) & 107.42 & 4 & ASN705 (2.79), ARG752 (2.85), THR877 (2.87) \\
\hline AR (1E3G) & Metribolone (Crystal structure) & - & 3 & GLN711 (2.62), ARG752 (1.72 and 2.95), THR877 (2.33) \\
& Metribolone (Docked pose) & 112.31 & 4 & THR877 (3.40) \\
& CPA (Docked pose) & 110.47 & 1 & GLN711 (2.81 and 3.42), ARG752 (2.14 and 3.20), \\
& Piperine (Docked pose) & 106.81 & 5 & THR877 (3.22)
\end{tabular}

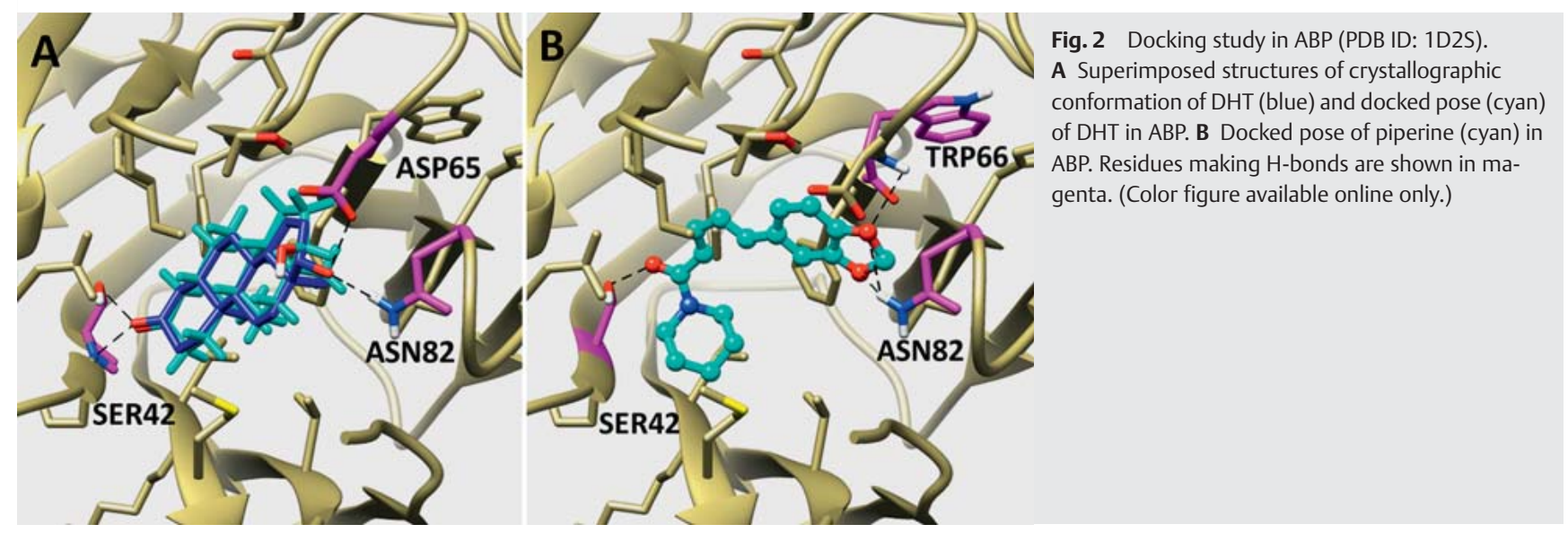

the crystallographic conformation of the ligand present in $1 \mathrm{E} 3 \mathrm{G}$, with an RMSD of $3.0 \AA$, between the two conformations. Metribolone interacted with AR by making four H-bonds with three residues, Gln711, Arg752, and Thr877 ( Fig.3A and Table 1). Hbond interactions with Arg752 and Thr877 were also present in the cocrystal complex of metribolone-AR. However, an H-bond interaction with Asn705 was not observed in the docked pose. CPA, a well-known antagonist of AR, was included in this study to compare the agonist and antagonist interactions with the target AR. CPA docked to AR with a score of 110.47 and interacted by making one $\mathrm{H}$-bond with Thr877 ( Fig. 3 B and Table 1). The presence of $\mathrm{H}$-bonds between the ligand and the protein were determined with a distance cutoff of $<3.5$ Á.

Next, piperine was docked to the active site of the ABP and found to bind to the protein with the top pose having a LibDock score of 107.42 ( Table 1). A total of 92 poses for piperine was generated. To assess the reproducibility and reliability of the piperine docking results, the experiment was repeated two more times. It was found that the piperine docked to the target protein with the same LibDock score of 107.42 and produced the same number of piperine poses in these experiments. In all three docking experiments, the top pose had a similar conformation, with an RMSD between the poses $<0.21 \AA$, suggesting that the predicted pose of piperine in $A B P$ is reproducible and reliable. Piperine interacted with $\mathrm{ABP}$ by making four $\mathrm{H}$-bonds with three amino acid residues, Ser42, Trp66, and Asn82 ( Fig. 2B and Table 1). The amide carbonyl group of piperine interacted with the Ser $42 \mathrm{OH}$ group, and the oxygen atom of the dioxalane ring interacted with the main chain NH group of Trp66 and the side chain of Asn82. This shows that piperine interacted with $\mathrm{ABP}$ in a manner similar to that of DHT, as interactions with Ser42 and Asn82 were also present in the crystal structure complex of DHT-ABP.

The docking of piperine to AR showed that it was able to dock to the active site of AR with the top pose showing a LibDock score of 106.81 ( Table 1). A total of 42 poses for piperine was generated. The docking experiments were repeated two more times, which showed that the LibDock score did not alter between the experiments. The top docked pose of piperine was similar in all three experiments, with an RMSD between the poses $<0.22 \AA$. Piperine interacted with AR by making five $\mathrm{H}$-bonds with three residues, Gln711, Arg752, and Thr877 ( Fig. 3C and Table 1). The amide carbonyl group of piperine made one H-bond with the Thr877 backbone $\mathrm{NH}$ group and the two oxygen atoms of a dioxalane ring made four $\mathrm{H}$-bonds with a Gln711 backbone $\mathrm{NH}$ and Arg752 side chain $\mathrm{NH}_{2}$ group. The docking results show that piperine interacted with AR in a manner similar to that of the agonist metribolone and antagonist CPA. The interaction with Arg752 and Thr877 was also present in the crystal structure complex of metribolone-AR, and the interaction with Thr877 was present in the case of CPA-AR.

In addition to analyzing the top scoring pose of piperine in $A B P$ and $A R$, all the generated poses (92 in ABP and 42 in AR) were clustered based on RMSD (cutoff of $2 \AA$ ) and the top scoring pose from each cluster was analyzed for interactions with the target. Piperine in ABP produced a total of ten clusters from 92 docked poses (Table 1S and Fig. 1S, Supporting Information). The top scoring pose from five clusters showed an H-bond interaction with the important Ser42 residue; whereas, interactions with Asn82 were observed in all of the top poses from the ten clusters. Similar clustering analysis showed that in AR, piperine produced five clusters from 42 docked poses (Table 1S and Fig. 2S, Support- 


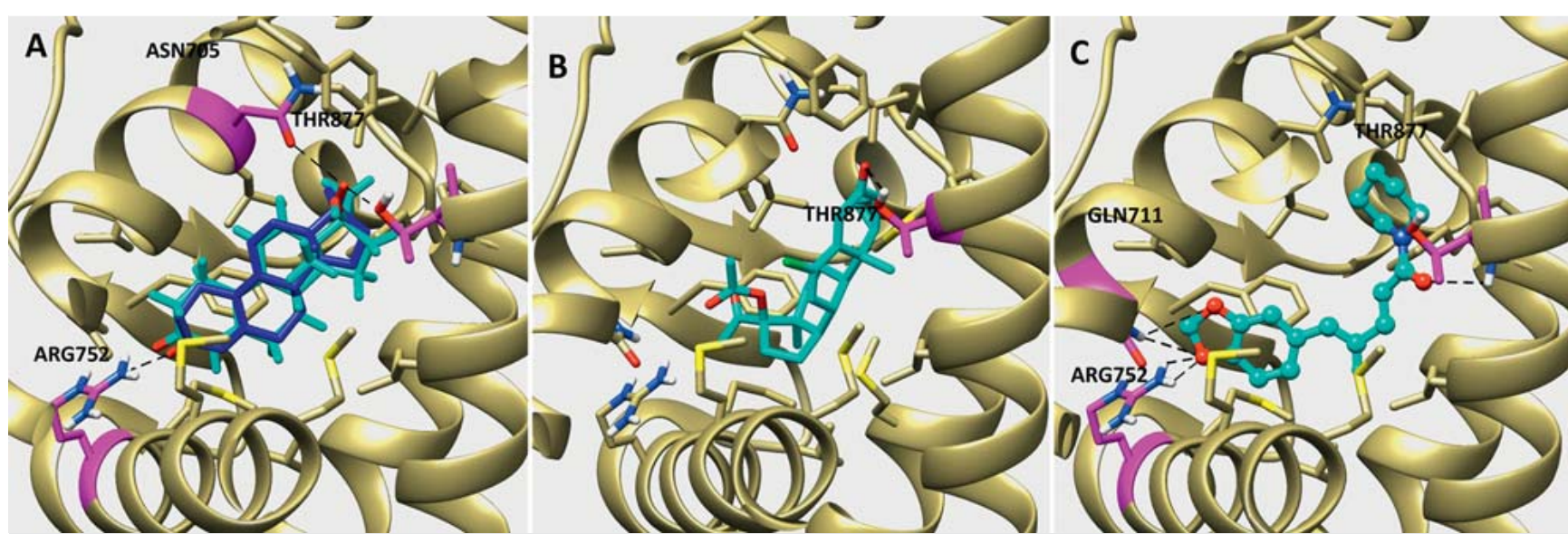

Fig. 3 Docking study in AR (PDB ID: 1E3G). A Superimposed structures of crystallographic conformation of metribolone (blue) and docked pose (cyan) of metribolone. Docked pose of CPA (B) and piperine (C) in AR. Residues making $\mathrm{H}$-bonds are shown in magenta. (Color figure available online only.) ing Information). Out of them, the top scoring pose from three clusters maintained an $\mathrm{H}$-bond interaction with the key residues Arg752 and Thr877. Clustering analysis of the docked poses suggests that the key H-bond interactions of piperine with the target protein, as discussed above for the top scoring pose in ABP and $\mathrm{AR}$, are also present in other poses with a lower score.

To determine the stability of the predicted interaction by docking, each of the three docked complexes of piperine-ABP and piperine-AR were subjected to MD simulation individually for 10 ns. In MD simulation experiments, the physical movement of atoms and molecules is allowed, with a concomitant interaction with each other for a given period of time. Then the trajectory of interacting atoms and molecules is calculated by solving Newton's law of motion, whereas the forces between atoms and molecules and potential energy are calculated by a force field. MD simulation could help us to determine if the interaction between the protein and ligand is stable, such as the molecular interaction predicted for piperine with ABP and AR in the docking experiments.

For this purpose, the docked complexes (piperine-ABP and piperine-AR) were placed in a cubic box containing water molecules and simulated for $10 \mathrm{~ns}$. The stability of the complex was evaluated by calculating the protein backbone RMSDs and RMSFs of individual amino acid residues during the simulation with respect to the initial structure. The presence of $\mathrm{H}$-bonds between the protein residues and the ligand, their percentage of existence as well as potential energy (PE) of the protein-ligand complex were monitored.

Analysis of the RMSD graph of the three piperine-ABP complexes showed that the system reached an equilibrium condition within 100 ps of the simulation period and had a low $\operatorname{RMSD}(<2.5 \AA)$ value throughout the simulation period ( Fig. $4 \mathrm{~A}$ ). The average RMSD during the final $2 \mathrm{~ns}$ ( 8 to $10 \mathrm{~ns}$ ) of simulation was in the range of 1.9 to $2.3 \AA$, suggesting that all three piperine-ABP complexes behaved quite similarly. Analysis of the RMSF graph ( Fig. 4B) showed that the terminal and the loop region residues were highly flexible; however, the active site residues such as Ser42 and Thr60 were less flexible in all the three piperine-ABP complexes.

Analysis of the $\mathrm{H}$-bonds formed during the simulation shows that each of the three complexes formed on an average of 1.17, 1.36, and $1.49 \mathrm{H}$-bonds per time frame ( Fig. $4 \mathrm{C}$ and Table 2). H-bond interaction with Ser42 was found to have a maximum occupancy of $88.4,84.0$, and $67.0 \%$ during the three simulations, respectively. Besides this, H-bonds with Thr60 (13.8\%) and Leu171 (29.3\%) were also observed, but the interaction was not present in all three simulations. However, two other the H-bond interactions predicted in the docking studies (Thr66 and Asn82) were not observed or the occupancy was less than $5 \%$ during the simulation in all three experiments. The simulation results show that piperine can make a stable interaction with the protein through the interaction with Ser42 residue. In ABP, Ser42 is conserved among several species and mutating Ser42 with leucine leads to a loss of steroid-binding ability, making Ser42 an important residue in the active site of ABP. The ability of piperine to interact stably with Ser42 in the active site of ABP could have important consequences to the ability of $\mathrm{ABP}$ to interact with testosterone, as discussed below. Also, in all three simulations, the protein-ligand complex energy $(-6.47325 \mathrm{e}+05 \mathrm{KJ} / \mathrm{mol},-6.47349 \mathrm{e}+05 \mathrm{KJ} / \mathrm{mol}$ and $-6.47341 \mathrm{e}+05 \mathrm{KJ} / \mathrm{mol}$ ) was stable throughout the simulation without much variation.

Further, to confirm the stability of the interactions of piperine with $\mathrm{ABP}$, one of the ABP-piperine complex simulations was extended up to $30 \mathrm{~ns}$ (Fig. 3S, Supporting Information). The findings are in line with the $10 \mathrm{~ns}$ simulation results, further confirming that the interactions between piperine and ABP are stable.

To understand the importance of the above observation, the DHT-ABP complex was subjected to a $10 \mathrm{~ns}$ simulation using the conditions employed for the piperine-ABP complex simulation. The RMSD, RMSF, and H-bond graphs for the simulation are shown in Fig. 4S, Supporting Information. The complex stabilized with an average RMSD of $1.73 \AA$ A during the last $2 \mathrm{~ns}$ ( 8 to $10 \mathrm{~ns}$ ) of the simulation. DHT, on average, formed a $1.4 \mathrm{H}$-bond with Ser42 during the simulation period, making a stable interaction $(77.7 \%)$ throughout the simulation period ( $\bullet$ Table 2 ). The average energy of the DHT-ABP complex during the simulation was $-6.472750 \mathrm{e}+05 \mathrm{KJ} / \mathrm{mol}$, which is slightly higher than the piperine-ABP complex energy $(-6.473383 e+05 \mathrm{KJ} / \mathrm{mol}$, an average from three simulations). This further supports the fact that piperine is interacting with ABP in a manner similar to that of DHT and can produce a male contraceptive effect by blocking the interaction of ABP with the natural ligands (DHT/testosterone).

Next, MD simulation of the piperine-AR complex was investigated. RMSD of the protein backbone atoms of AR showed stabi- 


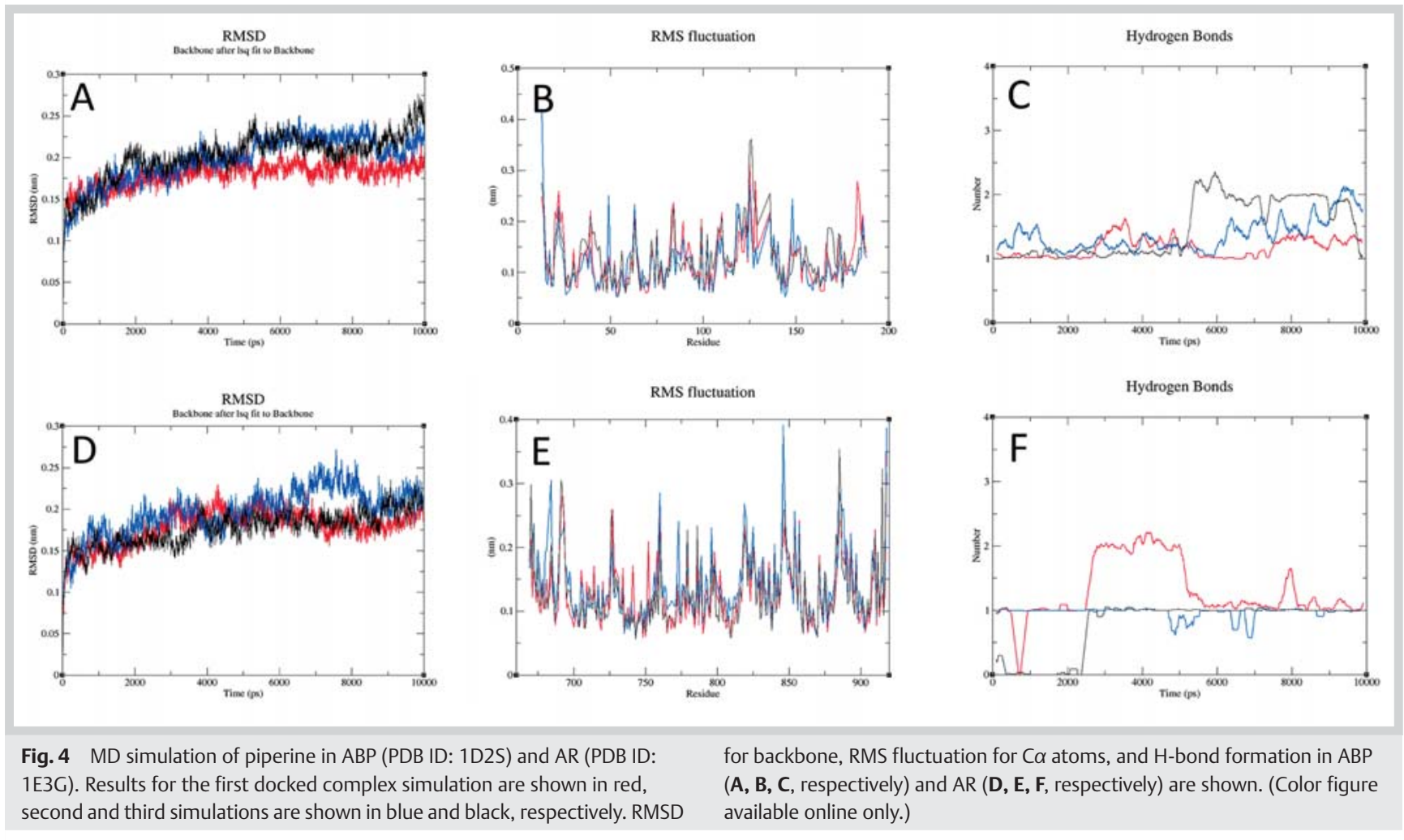

Table 2 Comparison of MD simulation (10 ns) results of piperine and DHT (native ligand) in ABP (PDB ID: 1D2S).

\begin{tabular}{|c|c|c|c|c|c|c|}
\hline \multirow{3}{*}{$\begin{array}{l}\text { Interacting } \\
\text { residue }\end{array}$} & \multirow{3}{*}{$\begin{array}{l}\text { H-bond in } \\
\text { DHT-ABP crystal } \\
\text { structure }\end{array}$} & \multirow{3}{*}{$\begin{array}{l}\mathrm{H} \text {-bond in } \\
\text { Piperine-ABP } \\
\text { docking }\end{array}$} & \multicolumn{4}{|c|}{ \% Occupancy of $\mathrm{H}$-bond in MD simulation } \\
\hline & & & \multirow[t]{2}{*}{ DHT-ABP } & \multicolumn{3}{|c|}{ Piperine-ABP } \\
\hline & & & & 1 & 2 & 3 \\
\hline SER42 & Yes & Yes & 77.7 & 88.4 & 84.0 & 67.0 \\
\hline THR60 & No & No & 1.0 & 4.3 & 13.8 & - \\
\hline ASP65 & Yes & No & 2.5 & - & - & - \\
\hline TRP66 & No & Yes & - & - & - & - \\
\hline ASN82 & Yes & Yes & 17.7 & 0.5 & 1.8 & - \\
\hline SER128 & No & No & 0 & 6.0 & 0 & - \\
\hline LEU171 & No & No & - & - & 0 & 29.3 \\
\hline \multicolumn{3}{|c|}{ Average $\mathrm{H}$-bonds per time frame } & 1.41 & 1.17 & 1.36 & 1.49 \\
\hline
\end{tabular}

lization within $100 \mathrm{ps}$ of the simulation period in the three systems, with a maximum RMSD of $2.5 \AA$ throughout the simulation period ( $\odot$ Fig. 4D). The average RMSD for the three complexes during the final $2 \mathrm{~ns}$ ( 8 to $10 \mathrm{~ns}$ ) of simulation were 1.9, 2.1, and 2.0 Å. Moreover, similar to the piperine-ABP complex, analysis of RMSF graphs of piperine-AR ( $\odot$ Fig. $4 \mathrm{E}$ ) showed that the terminal and the loop region residues were highly flexible. However, the active site residues, such as Asn705 and Thr877, were less flexible during the simulation in all three complexes. This suggests that all three piperine-AR complexes behaved similarly in the simulation experiments.

In addition, $\mathrm{H}$-bond formation during the simulation of piperineAR was analyzed in all three simulation experiments ( $\mathbf{O}$ Fig. $\mathbf{4 F}$ and Table 3). It showed that each of the three complexes formed on an average of $1.29,0.97$, and $0.77 \mathrm{H}$-bonds per time frame. Among the three simulations, H-bond interaction with Thr877 was found to have a maximum occupancy of $74.9,49.6$, and $97.8 \%$ during the simulation. In addition to this, $\mathrm{H}$-bonds with
Asn705 (18.9 and 50.0\%) were also observed in two out of three simulations. The Thr877 interaction was predicted in the docking studies, however, the other two $\mathrm{H}$-bond interactions predicted by docking, Gln711 and Arg752, were not observed or the occupancy was less than $6 \%$ during all three simulations. Also, in all three simulations, the protein-ligand complex energy $(-1.019040 \mathrm{e}+06 \mathrm{KJ} / \mathrm{mol}, \quad-1.018960 \mathrm{e}+06 \mathrm{KJ} / \mathrm{mol}$, and $-1.018980 \mathrm{e}+06 \mathrm{KJ} / \mathrm{mol}$ ) was stable throughout the simulation without much variation.

Further, to confirm the stability of the interactions of piperine with AR, one of the AR-piperine complex simulations was extended up to 30 ns (Fig. 5S, Supporting Information). The results show that the findings from the $30 \mathrm{~ns}$ simulation are in line with the 10 ns simulation results, further confirming that the interactions between piperine and $\mathrm{ABP}$ are stable.

Besides MD simulation of the piperine-AR complex, metriboloneAR and CPA-AR complexes were also simulated for $10 \mathrm{~ns}$ to understand the essential protein-ligand interactions in AR for ag- 
Table 3 Comparison of MD simulation (10 ns) results of piperine, metribolone (agonist), and CPA (antagonist) in AR (PDB ID: 1E3G).

\begin{tabular}{|c|c|c|c|c|c|c|c|}
\hline \multirow{3}{*}{$\begin{array}{l}\text { Interacting } \\
\text { residue }\end{array}$} & \multirow{3}{*}{$\begin{array}{l}\text { H-bond in } \\
\text { Metribolone-AR } \\
\text { crystal structure }\end{array}$} & \multirow{3}{*}{$\begin{array}{l}\text { H-bond in } \\
\text { Piperine-AR } \\
\text { docking }\end{array}$} & \multicolumn{5}{|c|}{ \% Occupancy of $\mathrm{H}$-bond in MD simulation } \\
\hline & & & Metribolone- & Cyproterone & Piperine-AR & & \\
\hline & & & AR & acetate-AR & 1 & 2 & 3 \\
\hline ASN705 & Yes & No & 1.0 & 18.5 & 18.9 & 50.0 & - \\
\hline GLN711 & No & Yes & 89.3 & 21.2 & 5.9 & 0 & 1.0 \\
\hline ARG752 & Yes & Yes & 7.5 & 0 & 0.2 & 0 & 1.0 \\
\hline THR877 & Yes & Yes & 2.5 & 60.2 & 74.9 & 49.6 & 97.8 \\
\hline \multicolumn{3}{|c|}{ Average $\mathrm{H}$-bonds per time frame } & 0.93 & 1.60 & 1.29 & 0.97 & 0.77 \\
\hline
\end{tabular}

onist and antagonist activity. RMSD, RMSF, and H-bond graphs for the metribolone-AR simulation are shown in Fig. 6S, Supporting Information. During the simulation, the metribolone-AR complex made a stable interaction with an average RMSD of $1.66 \AA$ during the last $2 \mathrm{~ns}$ ( 8 to $10 \mathrm{~ns}$ ) of the simulation. The average energy of the complex was $-1.018890+06 \mathrm{KJ} / \mathrm{mol}$, which is slightly higher than that of the piperine-AR complex energy of $-1.018993 e+06 \mathrm{KJ} / \mathrm{mol}$ (average from three simulations) during the simulation. Metribolone, on average, formed a $0.93 \mathrm{H}-$ bond during the simulation period, with Gln711 making a stable interaction (89.3\%) throughout the simulation period ( Table 3). However, other interactions present in the metribolone-AR crystal structure, Asn705, Arg752, and Thr877, had a low occupancy $(<10 \%)$ during the simulation. It should be noted that in the crystal structure complex of metribolone-AR (PDB ID: 1E3G), the Gln711 residue is not within the H-bond forming distance $(3.5 \AA$ cutoff) with the ligand, but during the simulation it was the major H-bond forming residue with the ligand.

The antagonist CPA also made a stable interaction with AR during the MD simulation, with an RMSD of $3.14 \AA$ A during the last 2 ns ( 8 to $10 \mathrm{~ns}$ ) of the simulation (Fig. 75, Supporting Information). The average energy of the CPA-AR complex was $-1.019330 \mathrm{e}+06 \mathrm{KJ} /$ mol, and the ligand, on average, made $1.6 \mathrm{H}$-bonds with the protein during the simulation. CPA made H-bonds with Asn705, Gln711, Arg752, and Thr877 residues. However, the H-bond interaction with the residue Thr877 had the best occupancy (60.22\%) during the simulation period ( Table 3). Piperine also made a stable $\mathrm{H}$-bond interaction with this important residue during the simulation, suggesting that piperine could act as an antagonist of AR, similar to the known CPA.

The MD simulation results of the piperine-AR complex shows that piperine is able to make a stable interaction with the target AR protein through the H-bond interaction with the Thr877 residue; however, the interaction between the piperine-AR complex is different than that of metribolone-AR. Among the nuclear receptor family proteins, the AR and progesterone receptor (PR) share similar structures. The specificity of the ligand binding to AR is defined by the presence of Thr877 in the active site region of AR and, hence, has a structural importance in the AR [39]. The ability of piperine to interact stably with Thr877 in the active site of AR suggests that piperine is capable of specifically interacting with AR and producing its male contraceptive effect by blocking the interaction of AR with its natural ligands (DHT/testosterone). Based on the in silico results, piperine was tested for its ability to antagonize DHT at AR in the MDA-kb2 cell line with the luciferase reporter gene assay. The MDA-kb2 cell line stably expresses androgen and glucocorticoid receptors, and we have used it to study the effect of endocrine-disrupting chemicals $[42,43]$ Initially, the maximum nontoxic concentration of piperine was determined by the CellTiter $96{ }^{\circledR}$ Aqueous One Solution Cell Proliferation As- say. Cells were incubated for $24 \mathrm{~h}$ with the androgen agonist DHT $(0.5 \mathrm{nM})$ and piperine at seven different concentrations $(150 \mu \mathrm{M}-20 \mu \mathrm{M})$. The metabolic activity was measured as described in the experimental section and is an indicator of cytotoxicity to the cells. Piperine was found to be cytotoxic at concentrations greater than $20 \mu \mathrm{M}$ (Fig. 8S, Supporting Information), and this was used as the maximum concentration in the luciferase reporter gene assay.

Various concentrations $(20 \mu \mathrm{M}$ to $0.01 \mathrm{nM})$ of piperine in the MDA-kb2 cell line treated with DHT $(0.5 \mathrm{nM})$ produced a very characteristic response ( Fig.5). An initial slight increase in transcriptional (luciferase) activity was observed up to a concentration of $1 \mu \mathrm{M}$ of piperine treatment. At concentrations below $1 \mu \mathrm{M}$, piperine significantly decreased the DHT-induced AR-mediated transcriptional activity. However, it did not show a linear dose-effect relationship. The existence of nonlinear and nonmonotonic effects is not unusual for receptor-binding endocrine disrupting substances $[44,45]$. Flutamide $\left(\mathrm{IC}_{50}=4.6 \mu \mathrm{M}\right)$, a known antagonist of the AR, was used as a positive control in the study. The in vitro assay results show that piperine possesses an antiandrogenic effect, as predicted in the in silico experiments.

Unlike for females, oral contraceptives are not available for males. Currently, the use of condoms or a vasectomy is the only choice available for male contraception. Hence, there is a need to develop reversible and safe oral contraceptives for males. Piperine, an active constituent of the Piper species, is known to possess a male contraceptive effect. However, how piperine acts as a male contraceptive is not very clear. Here, using in silico tools and an in vitro assay, the ability of piperine to interact with two targets, ABP and AR, was investigated to understand the molecular mechanism of action.

AR is essential for the development of a male phenotype and also controls male sexual behavior and fertility. The binding of the natural ligands testosterone and dihydrotestosterone to the AR induces a conformational change in the receptors that leads to the translocation of the AR to the nucleus, which results in the transcriptional regulation of androgen target genes. ABP binds testosterone and keep its concentration sufficient to produce spermatogenesis in testis. Docking and molecular dynamics simulations performed in this study suggest that piperine is able to interact stably with AR, similar to the antagonist CPA, and could block the interaction of natural ligands with AR. Also, piperine is able to make a stable interaction with $A B P$ and could act as a competitive antagonist for the binding of androgens. The interaction of piperine with AR and ABP could form the basis for piperine's male contraceptive effect.

Several modern drugs have been developed from Ayurveda and TCM, and have marketed the world over. To develop drugs based on traditional medicine, it is essential to understand the molecular mechanism of action of these drugs. In silico and in vitro stud- 


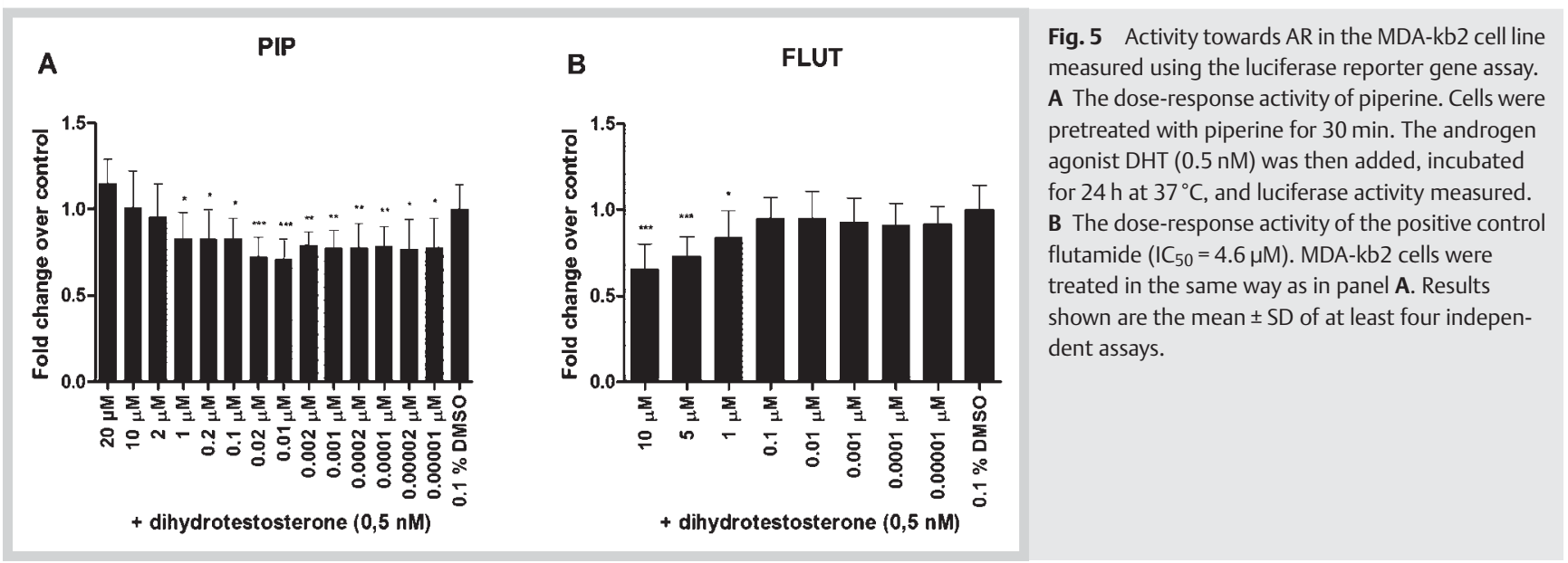

ies performed here show that piperine's male contraceptive effect could be due to its ability to interact with ABP and AR. As a next step in the search of a male contraceptive agent, detailed biochemical experiments need to be performed to unlock the true potential of piperine.

\section{Materials and Methods \\ $\nabla$}

\section{In silico studies}

Docking studies were performed with the LibDock module [46] of Discovery Studio v3.2. [47], and GROMACS 4.5.7 [48] was used for molecular dynamics simulations.

Preparation of the target protein and ligand structures: Structures of ABP (PDB ID: 1D2S) and AR (PDB ID: 1E3G) were retrieved from the protein database (www.rcsb.org) and prepared using the protein preparation module of Discovery Studio. Water molecules were removed from the protein and protonated at $\mathrm{pH}$ 7.4. The dielectric constant was set as 10 . Then, the protein structures were minimized with the CHARMm force field. The ligand structure was drawn using Marvin Sketch, saved in an sdf file format, and subjected to ligand preparation in Discovery Studio. The ligand structure was ionized between pH 6.5 and 8.5. This allowed for the generation of all possible tautomers and isomers. Discovery Studio generated only one output structure for piperine, which was used for docking with the target proteins.

Docking: The active site for docking was identified based on the crystallized ligand present in the protein. ABP (PDB ID: 1D2S) has DHT, and AR (PDB ID: 1E3G) has metribolone as the ligands. In the docking experiments, the target protein was kept rigid and the ligand was flexible. The active site sphere was set around the known crystal structure ligands with a radius of 6.08 Á and $6.29 \AA$ for $1 \mathrm{D} 2 \mathrm{~S}$ and $1 \mathrm{E} 3 \mathrm{G}$, respectively. The docking of piperine with the target protein was performed using the LibDock module of Discovery Studio with the following settings: The best conformation method was selected for high-quality results and the number of hotspots was set to a maximum of 100 for the docking calculations. Docking tolerance was set to 0.25 . For validation of the docking procedure, known crystal structure ligands along with piperine were included in the docking study. The pose with the maximum LibDock score was considered the best fitting pose and the complex with the pose was taken for MD simulations. Molecular dynamics simulations: For the simulations, Gromos43a1 force field was used. Ligand topology files were generated using the PRODRG server (http://davapc1.bioch.dundee.ac.uk/ cgi-bin/prodrg). Then, the protein-ligand complex was solvated in a cubic box ( $1.5 \mathrm{~nm}$ ) with SPC water molecules [49]. Neutralization of the system was done by adding $\mathrm{Cl}^{-}$or $\mathrm{Na}^{+}$ions. The setup was equilibrated at $300 \mathrm{~K}$ for $200 \mathrm{ps}$, before the MD production run. Then MD production run was done for $10 \mathrm{~ns}$ and the trajectories were written for every 2 fs. Default GROMACS criteria were used for the analysis of the trajectory.

\section{In vitro studies}

Chemicals: DHT ( $\geq 97.5$ pure, CAS No. 521-18-6) and flutamide (FLUT, $\geq 99$ pure, CAS No.13311-84-7) were from Sigma-Aldrich. Piperine ( $\geq 95$ pure, CAS No. 94-62-2) was purchased from Sigma-Aldrich. Compounds were dissolved in dimethyl sulfoxide (DMSO, 99.9\%, Sigma-Aldrich).

Cell culture and assay of viability: The MDA-kb2 cell line (ATCC), stably transformed with murine mammalian tumor virus (MMTV)-luciferase, was cultured in Leibovitz's L-15 medium with $10 \%$ fetal bovine serum (Gibco), $100 \mathrm{U} / \mathrm{mL}$ penicillin (Sig$\mathrm{ma}$ ), and $100 \mu \mathrm{g} / \mathrm{mL}$ streptomycin (Sigma) at $37^{\circ} \mathrm{C}$ without $\mathrm{CO}_{2}$. For the experiments, cells were seeded at $1 \times 105$ cells $/ \mathrm{mL}$ $(100 \mu \mathrm{L} /$ well $)$ in 96 -well microtiter plates (TPP). After the cells were attached $(24 \mathrm{~h})$, the medium was removed and replaced with the compound of interest dissolved in medium or the corresponding vehicle (control wells). After $24 \mathrm{~h}$ incubation, the CellTiter 96 ${ }^{\circledR}$ Aqueous One Solution Cell Proliferation Assay (Promega) was used to evaluate metabolic activity. Absorbance was measured at $492 \mathrm{~nm}$ by the automatic microplate reader Synergy $^{\mathrm{TM}} 4$ Hybrid Microplate Reader (BioTek).

Luciferase reporter gene assay: For reporter gene experiments, cells were collected from culture dishes and seeded at a density of $1 \times 105$ cells/mL $(100 \mu \mathrm{L} /$ well $)$ in 96-well luminometer plates (Greiner Bio-One $\mathrm{GmbH})$. When the cells were attached $(24 \mathrm{~h})$, the medium was removed and replaced with medium containing the compound of interest. The compound was tested at nontoxic concentrations. After $24 \mathrm{~h}$ incubation, the cells were rinsed three times with phosphate-buffered saline (PBS), and luciferase activity was determined with the luciferase reporter assay system kit (Promega) following the manufacturer's instructions. Luminescence was measured with the automatic microplate reader Synergy $^{\mathrm{TM}} 4$ Hybrid Microplate Reader (BioTek). The relative transcriptional activity was converted to fold induction above the corresponding vehicle control value ( $\mathrm{n}$-fold). 
Data and statistical analysis: Results are expressed as means \pm SD from at least four independent experiments with at least duplicate wells for each treatment. Statistical significance was tested by one-way analysis of variance (ANOVA), followed by StudentNewman-Keuls test, where ${ }^{*} \mathrm{p}<0.05,{ }^{* *} \mathrm{p}<0.01,{ }^{* * *} \mathrm{p}<0.001$ were considered statistically significant.

\section{Supporting information}

Clustering analysis results of the docked poses of piperine in the $A B P$ and $A R$, molecular dynamics simulation results of AR-piperine and ABP-piperine for $30 \mathrm{~ns}$ each, and AR-metribolone, AR-C$\mathrm{PA}$, and ABP-DHT for $10 \mathrm{~ns}$ each, and the cytotoxicity effect of piperine are provided.

\section{Acknowledgements}

$\nabla$

G.C., L.P., and M.S.C. thank the Department of Biotechnology (DBT), Government of India, for financial assistance to the DBTIPLS program, Pondicherry University. M.S.C. also thanks DBT for providing financial assistance (DBT's Twining program for North East-BT/246/NE/TBP/2011/77) to purchase the server. Also, the authors thank Prof. K. Srikumar, Coordinator, DBT-IPLS program, Pondicherry University.

\section{Conflict of Interest}

The authors declare no conflict of interest.

\section{Affiliations}

${ }^{1}$ Interdisciplinary Program in Life Sciences, Pondicherry University, Kalapet, Puducherry, India

${ }^{2}$ Centre for Bioinformatics, School of Life Sciences, Pondicherry University, Kalapet, Puducherry, India

3 Faculty of Pharmacy, University of Ljubljana, Ljubljana, Slovenia

${ }^{4}$ Department of Biochemistry and Molecular Biology, School of Life Sciences, Pondicherry University, Kalapet, Puducherry, India

\section{References}

1 Dias DA, Urban S, Rosenner U. A historical overview of natural products in drug discovery. Metabolites 2012; 2: 303-306

2 Ji HF, Li XJ, Zhang HY. Natural products and drug discovery. Can thousands of years of ancient medical knowledge lead us to new and powerful drug combinations in the fight against cancer and dementia? EMBO Rep 2009; 10: 194-200

3 Corson TW, Crews CM. Molecular understanding and modern application of traditional medicines: triumphs and trials. Cell 2007; 130: 769-774

4 Newman DJ, Cragg GM, Snader KM. Natural products as sources of new drugs over the period 1981-2002. J Nat Prod 2003; 66: 1022-1037

5 Srinivasan $K$. Black pepper and its pungent principle-piperine: a review of diverse physiological effects. Crit Rev Food Sci Nutr 2007; 47: 735-748

6 Meghwal M, Goswami TK. Piper nigrum and piperine: an update. Phytother Res 2013; 27: 1121-1130

7 Butt MS, Pasha I, Sultan MT, Randhawa MA, Saeed F, Ahmed W. Black pepper and health claims: a comprehensive treatise. Crit Rev Food Sci Nutr 2013; 53: 875-886

8 Li S, Wang C, Wang M, Li W, Matsumoto K, Tang Y. Antidepressant like effects of piperine in chronic mild stress treated mice and its possible mechanisms. Life Sci 2007; 80: 1373-1381

9 Wattanathorn J, Chonpathompikunlert P, Muchimapura S, Priprem A, Tankamnerdthai $O$. Piperine, the potential functional food for mood and cognitive disorders. Food Chem Toxicol 2008; 46: 3106-3110

10 Park BS, Son DJ, Park YH, Kim TW, Lee SE. Antiplatelet effects of acidamides isolated from the fruits of Piper longum L. Phytomedicine 2007; 14: 853-855
11 Panda S, Kar A. Piperine lowers the serum concentrations of thyroid hormones, glucose and hepatic 5'D activity in adult male mice. Horm Metab Res 2003; 35: 523-526

12 Koul IB, Kapil A. Evaluation of the liver protective potential of piperine, an active principle of black and long peppers. Planta Med 1993; 59: 413-417

13 Atal S, Agrawal RP, Vyas S, Phadnis P, Rai N. Evaluation of the effect of piperine per se on blood glucose level in alloxan-induced diabetic mice. Acta Pol Pharm 2012; 69: 965-969

14 Mujumdar AM, Dhuley JN, Deshmukh VK, Raman PH, Naik SR. Anti-inflammatory activity of piperine. Jpn J Med Sci Biol 1990; 43: 95-100

15 Samykutty A, Shetty AV, Dakshinamoorthy G, Bartik MM, Johnson GL, Webb B, Zheng G, Chen A, Kalyanasundaram R, Munirathinam G. Piperine, a Bioactive Component of Pepper Spice Exerts Therapeutic Effects on Androgen Dependent and Androgen Independent Prostate Cancer Cells. PLoS One 2013; 8: e65889

$16 \mathrm{Kim}$ SH, Lee YC. Piperine inhibits eosinophil infiltration and airway hyperresponsiveness by suppressing $\mathrm{T}$ cell activity and Th2 cytokine production in the ovalbumin-induced asthma model. J Pharm Pharmacol 2009; 61: 353-359

17 Veerareddy PR, Vobalaboina $V$, Nahid A. Formulation and evaluation of oil-in-water emulsions of piperine in visceral leishmaniasis. Pharmazie 2004; 59: 194-197

18 El Hamss R, Idaomar M, Alonso-Moraga A, Muñoz Serrano A. Antimutagenic properties of bell and black peppers. Food Chem Toxicol 2003; 41: $41-47$

19 Bajad S, Bedi KL, Singla AK, Johri RK. Antidiarrhoeal activity of piperine in mice. Planta Med 2001; 67: 284-287

20 Kulkarni SK, Bhutani MK, Bishnoi M. Antidepressant activity of curcumin: involvement of serotonin and dopamine system. Psychopharmacology (Berl) 2008; 201: 435-442

21 Zhao JQ Du GZ, Xiong YC, Wen YF, Bhadauria M, Nirala SK. Attenuation of beryllium induced hepatorenal dysfunction and oxidative stress in rodents by combined effect of gallic acid and piperine. Arch Pharm Res 2007; 30: 1575-1583

22 Nirala SK, Bhadauria M, Mathur R, Mathur A. Influence of alpha-tocopherol, propolis and piperine on therapeutic potential of tiferron against beryllium induced toxic manifestations. J Appl Toxicol 2008; 28: 44-54

23 Badmaev V, Majeed M, Norkus EP. Piperine, an alkaloid derived from black pepper increases serum response of beta-carotene during 14days of oral beta-carotene supplementation. Nutr Res 1999; 19: 381388

24 Lambert JD, Hong J, Kim DH, Mishin VM, Yang CS. Piperine enhances the bioavailability of the tea polyphenol (-)-epigallocatechin-3-gallate in mice. J Nutr 2004; 134: 1948-1952

25 Patil UK, Singh A, Chakraborty AK. Role of piperine as a bioavailability enhancer. Int J Rec Adv Pharma Res 2011; 4: 16-23

26 Han HK. The effects of black pepper on the intestinal absorption and hepatic metabolism of drugs. Expert Opin Drug Metab Toxicol 2011; 7: 721-729

27 Atal N, Bedi KL. Bioenhancers: Revolutionary concept to market. J Ayurveda Integr Med 2010; 1: 96-99

28 Gupta RS, Sharma R. A review on medicinal plants exhibiting antifertility activity in males. Nat Prod Rad 2006; 5: 389-410

29 Malini T, Manimaran RR, Arunakaran J, Aruldhas MM, Govindarajulu P. Effects of piperine on testis of albino rats. J Ethnopharmacol 1999; 64: 219-225

30 Daware MB, Mujumdar AM, Ghaskadbi S. Reproductive toxicity of piperine in Swiss albino mice. Planta Med 2000; 66: 231-236

31 D'cruz SC, Mathur PP. Effect of piperine on the epididymis of adult male rats. Asian J Androl 2005; 7: 363-368

32 D'Cruz SC, Vaithinathan S, Saradha B, Mathur PP. Piperine activates testicular apoptosis in adult rats. J Biochem Mol Toxicol 2008; 22: 382388

33 Ruizeveld de Winter JA, Trapman J, Vermey M, Mulder E, Zegers ND, van der Kwast TH. Androgen receptor expression in human tissues: an immunohistochemical study. J Histochem Cytochem 1991; 39: 927-936

34 Holdcraft RW, Braun RE. Androgen receptor function is required in Sertoli cells for the terminal differentiation of haploid spermatids. Development 2004; 131: 459-467

35 Chang C, Lee SO, Wang RS, Yeh S, Chang TM. Androgen receptor (AR) physiological roles in male and female reproductive systems: lessons learned from AR-knockout mice lacking AR in selective cells. Biol Reprod 2013; 89: 21 
36 Collins LL, Lee HJ, Chen YT, Chang M, Hsu HY, Yeh S, Chang C. The androgen receptor in spermatogenesis. Cytogenet Genome Res 2003; 103: 299-301

37 Smith $L B$, Walker $W H$. The regulation of spermatogenesis by androgens. Semin Cell Dev Biol 2014; 30: 2-13

38 Hirawat S, Budman DR, Kreis W. The androgen receptor: structure, mutations, and antiandrogens. Cancer Invest 2003; 21: 400-417

39 Matias PM, Donner P, Coelho R, Thomaz M, Peixoto C, Macedo S, Otto N, Joschko S, Scholz P, Wegg A, Basler S, Schafer M, Egner U, Carrondo MA. Structural evidence for ligand specificity in the binding domain of the human androgen receptor. Implications for pathogenic gene mutations. J Biol Chem 2000; 275: 26164-26171

40 Avvakumov GV, Cherkasov A, Muller YA, Hammond GL. Structural analyses of sex hormone-binding globulin reveal novel ligands and function. Mol Cell Endocrinol 2010; 316: 13-23

41 Grishkovskaya I, Avvakumov GV, Sklenar G, Dales D, Hammond GL, Muller YA. Crystal structure of human sex hormone-binding globulin: steroid transport by a laminin G-like domain. EMBO J 2000; 19: 504-512

42 Kolšek K, Gobec M, Mlinaric Raščan I, Sollner Dolenc M. Screening of bisphenol A, triclosan and paraben analogues as modulators of the glucocorticoid and androgen receptor activities. Toxicol In Vitro 2015; 29: 8-15
43 Klopčič I, Kolšek K, Dolenc MS. Glucocorticoid-like activity of propylparaben, butylparaben, diethylhexyl phthalate and tetramethrin mixtures studied in the MDA-kb2 cell line. Toxicol Lett 2015; 232: 376-383

44 Vandenberg LN, Wadia PR, Schaeberle CM, Rubin BS, Sonnenschein C, Soto $A M$. The mammary gland response to estradiol: monotonic at the cellular level, non-monotonic at the tissue-level of organization? J Steroid Biochem Mol Biol 2006; 101: 263-274

45 De Coster S, van Larebeke N. Endocrine-disrupting chemicals: associated disorders and mechanisms of action. J Environ Public Health 2012; 2012: 713696

46 Rao SN, Head MS, Kulkarni A, LaLonde JM. Validation studies of the sitedirected docking program LibDock. J Chem Inf Model 2007; 47: 21592171

47 Accelrys Software Inc. Discovery Studio Modeling Environment. Release 4.0. San Diego: Accelrys Software Inc.; 2013

48 Hess B, Kutzner C, van Der Spoel D, Lindahl E. GROMACS 4: Algorithms for highly efficient, load-balanced, and scalable molecular simulation. J Chem Theory Comput 2008; 4: 435-447

49 Berdenson HJC, Postma JPM, Gunstreen WFV, Hermans J. Interaction models for water in relation to protein hydration. In: Pullman B, editor. Intermolecular forces. Dordrecht: Springer Netherlands; 1981: 331342 